\title{
Elastic anomalies at terahertz frequencies and excess density of vibrational states in silica glass
}

\author{
Giacomo Baldi* \\ IPCF-CNR, UOS di Roma, I-00185 Roma, Italy and Dipartimento di Fisica, Università di Parma, I-43124, Parma, Italy \\ Valentina M. Giordano \\ LPMCN, Université Claude Bernard Lyon 1 et CNRS, UMR 5586, F-69622 Villeurbanne, France
}

Giulio Monaco

European Synchrotron Radiation Facility, Bôite Postale 220, F-38043 Grenoble, France

(Received 28 November 2010; revised manuscript received 12 March 2011; published 11 May 2011)

\begin{abstract}
We study the temperature dependence of acousticlike excitations measured by means of inelastic x-ray scattering at terahertz frequencies in silica glass. The apparent sound velocity shows, between 300 and $1600 \mathrm{~K}$, the same temperature variation measured, at lower frequencies, by Brillouin light scattering. On the contrary the vibrations at the boson peak (BP) present a much stronger temperature dependence, as indicated by neutron scattering data. The measured dispersion and damping are used to compute the contribution to the vibrational density of states (VDOS) coming from the propagating acousticlike modes. This part of the VDOS accounts only for a fraction of the BP intensity, indicating that other kinds of excitation accumulate in this frequency range. It is consequently not surprising that the BP does not follow the temperature evolution of the Debye frequency, which describes the modification of the continuum medium. Finally we present a comparison between the experimentally accessible quantities and a recently proposed model for the vibrations in glasses, based on the assumption of random spatial variations of the shear modulus [Schirmacher, Europhys. Lett. 73, 892 (2006)].
\end{abstract}

DOI: 10.1103/PhysRevB.83.174203

\section{INTRODUCTION}

A peculiarity of glasses is the presence of an excess of vibrational states over the prediction of the Debye model. In the frequency range of a few terahertz the reduced density of vibrational states, $g(\omega) / \omega^{2}$, presents a maximum, which is usually called the boson peak (BP). ${ }^{1}$ This feature is ubiquitous in all glasses as revealed by inelastic neutron scattering or Raman spectroscopy. Although studied for many years, the nature of the modes associated with this peak is still a matter of lively debate. These modes are responsible for the anomalous low-temperature properties of glasses, ${ }^{2}$ a maximum in $C_{p} / T^{3}$, where $C_{p}$ is the specific heat, and a plateau in the thermal conductivity in the $\sim 10 \mathrm{~K}$ temperature range.

The absence of periodicity that characterizes amorphous solids makes it very difficult to determine the nature of the vibrational modes. This is particularly true for the states lying around the boson peak. This frequency range is also at the lower edge of the region accessible to molecular dynamics simulations. ${ }^{3}$ A variety of theoretical models has been proposed to explain these low-frequency $(\sim 1 \mathrm{THz})$ vibrations. Among them we may recall the soft potential model, ${ }^{4}$ the mode-coupling theory applied to the vibrations in glasses, ${ }^{5}$ models on a lattice ${ }^{6}$ or on the continuum ${ }^{7}$ with randomly fluctuating elastic constants, and harmonic models where the atoms vibrate around topologically disordered configurations. ${ }^{8,9}$

Experimentally the BP has been studied as a function of macroscopic parameters such as the temperature, ${ }^{10,11}$ the density, ${ }^{12-15}$ and the pressure ${ }^{16,17}$ or during chemical vitrification ${ }^{18}$ or as a function of the quenching rate. ${ }^{19} \mathrm{It}$ is observed that the BP shifts to higher frequencies and decreases in intensity when the pressure or the density is increased. However, controversial results have been reported on the connection between the BP modification and the change in the
PACS number(s): 63.50.Lm, 62.65.+k, 64.70.ph

elastic medium properties. In some systems, ${ }^{14,18,19}$ it appears that the BP variation is solely associated with the change in the Debye frequency, which accounts for the modifications of the elastic continuum medium. In other cases, ${ }^{13,15-17}$ the BP shifts more strongly than suggested by the simple variation of the macroscopic medium. Specifically, this second behavior is observed when the system is subjected to an important volume change, of the order of $10 \%$ to $20 \%$. In the prototypical glass of silica, the BP shows a temperature dependence much stronger than that of the Debye frequency, computed from the Brillouin light scattering data in the gigahertz range, as noted in Ref. 20. However, a proper determination of the Debye frequency requires a measurement of the sound velocity in the region of frequency of the BP, in order to eliminate the effects of relaxations or anharmonicities. ${ }^{21}$ This can only be accomplished by using the inelastic x-ray scattering (IXS) technique, which gives access to the Brillouin doublet in the terahertz frequency range.

By means of IXS it has been recently observed ${ }^{22-25}$ that the $\mathrm{BP}$ is associated with anomalies in the elastic properties of the glass. The apparent sound velocity shows a negative dispersion and the sound attenuation undergoes a transition from a lowfrequency region where it is proportional to the fourth power of the frequency to a quadratic frequency dependence at high frequencies. This behavior has been observed in three systems with different degree of fragility: the glass of glycerol ${ }^{22}$ silica glass $^{23,24}$ at elevated temperatures, and sorbitol glass. ${ }^{25}$ These experimental results have been confirmed by molecular dynamics simulations of a Lennard-Jones glass performed on an exceptionally large simulation box, containing $10^{7}$ particles. ${ }^{3}$ The observation of these elastic anomalies requires a very high accuracy which has been attained only in recent years, thanks to the continuous development of the IXS technique. The particular case of vitreous silica has been deeply studied 
by means of IXS in the past, ${ }^{26-35}$ but these fine features of the dynamics were hidden by the noise in the first experiments.

In this work we present additional IXS data of vitreous silica, measured in the glassy state at room temperature, discussed together with the high-temperature data presented in a previous Letter. $^{23}$ The variation of the sound velocity with temperature is found to be comparable to that determined by means of Brillouin light scattering (BLS). ${ }^{36}$ Using the resulting Debye frequency we show that the temperature dependence of the BP in silica does not follow the elastic medium transformation, confirming the observation of Ref. 20. A change in slope of the sound attenuation is observed also at room temperature and it is found to be strictly related to the BP. We then present a computation of the acoustic contribution to the density of states based on the measured dispersion and damping of the excitations. The result indicates that these modes account only for a fraction, although not negligible, of the BP. Finally we compare the experimental results with a recently proposed model for the vibrations in glasses. ${ }^{7,37}$

\section{EXPERIMENT AND DATA ANALYSIS}

The experiment was performed at the inelastic x-ray scattering beamline ID16 of the European Synchrotron Radiation Facility in Grenoble. A high-energy resolution of $1.3 \mathrm{meV}$ is achieved by means of a silicon crystal monochromator working in backscattering configuration at the $(12,12,12)$ reflection, corresponding to an incident photon energy of $23.7 \mathrm{keV}$. The monochromatic beam is focused on the sample to a spot size of 300 (horizontal) $\times 100$ (vertical) $\mu \mathrm{m}^{2}$ full width at half maximum. The scattered radiation is energy analyzed by a set of nine analyzer crystals, thus enabling the simultaneous collection of nine spectra at different exchanged wave vectors $Q$. The collection angle of the analyzers corresponds to a wave vector uncertainty $\Delta Q \sim 0.34 \mathrm{~nm}^{-1}$. The sample used in the experiment is a Suprasil glass cylinder of $3.5 \mathrm{~mm}$ in length, chosen to match the photoabsorption length at the incident $\mathrm{x}$-ray energy.

A selection of spectra is plotted in Fig. 1 as a function of the exchanged energy. The figure shows the comparison between the spectra measured at high temperature, $T=1620 \mathrm{~K}$ (presented in a recent Letter $^{23}$ ), and the spectra collected at room temperature. The temperature strongly affects the inelastic signal because of the Bose population factor. This is reflected in an inelastic intensity at room temperature of about $5 \%$ of the elastic one. Each spectrum is measured for a total integration time of about $35 \mathrm{~h}$.

The measured intensity is proportional to the convolution of the instrument response function $R(\omega)$ with the dynamic structure factor $S(Q, \omega)$, multiplied by a factor which accounts for the detailed balance between the Stokes and anti-Stokes sides: ${ }^{35}$

$$
I(Q, \omega)=y_{0}+A(Q) \int \frac{\beta \hbar \omega^{\prime}}{1-e^{-\beta \hbar \omega^{\prime}}} S\left(Q, \omega^{\prime}\right) R\left(\omega-\omega^{\prime}\right) d \omega^{\prime} .
$$

In this expression $y_{0}$ is a baseline accounting for the background noise, $A(Q)$ a proportionality factor, and $\beta=\left(k_{B} T\right)^{-1}$, with $k_{B}$ the Boltzmann constant and $T$ the temperature. In the one-phonon approximation the inelastic contribution to

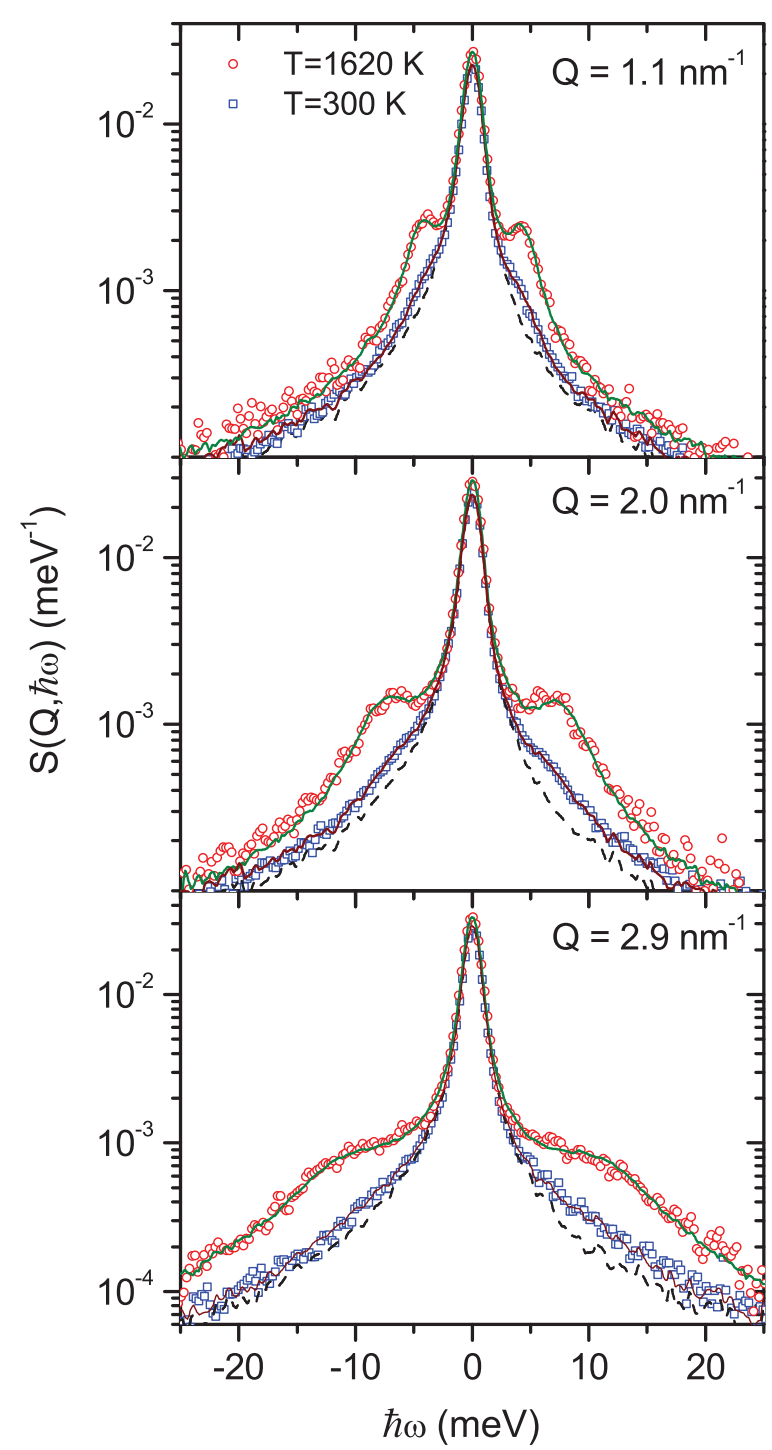

FIG. 1. (Color online) Selection of IXS spectra of vitreous silica at the indicated exchanged momenta $Q$. Each panel shows the spectra at two temperatures: $300 \mathrm{~K}$ (blue squares) and $1620 \mathrm{~K}$ (red circles). The best-fitting functions are plotted as continuous lines (wine at $300 \mathrm{~K}$ and olive at $1620 \mathrm{~K}$ ). The dashed black line is the elastic line, measured on a Plexiglass sample at $10 \mathrm{~K}$.

the dynamic structure factor can be written in terms of the longitudinal part $G_{L}(Q, z)$ of the resolvent operator of the dynamical matrix in the wave vector representation: ${ }^{38,39}$

$$
S_{i n}(Q, \omega)=-\frac{k_{B} T Q^{2}}{\pi m \omega} \lim _{\epsilon \rightarrow 0^{+}} \operatorname{Im} G_{L}\left(Q, \omega^{2}+i \epsilon\right),
$$

where $m$ is the average atomic mass. With great generality the phonon propagator can be expressed in terms of a complex self-energy $\Sigma(Q, z)$ :

$$
G_{j}(Q, z)=\frac{1}{z-v_{j, 0}^{2} Q^{2}+\Sigma_{j}(Q, z)} .
$$

Here the subscript $j=L, T$ indicates the longitudinal or the transverse component and $v_{j, 0}$ is the low-frequency limit of 
the corresponding sound velocity. Choosing for the self-energy the expression

$$
\Sigma(Q, \omega)=-Q^{2} \delta v^{2}(Q)+i \omega \Gamma(Q),
$$

the inelastic part of the dynamic structure factor reduces to a damped harmonic oscillator (DHO) peaked at $\Omega(Q)=$ $Q \sqrt{v_{0}^{2}+\delta v^{2}(Q)}$, where $\delta v^{2}$ is the variation of the square of the sound velocity, and with a full width at half maximum $\Gamma(Q)$. The DHO function is known to describe well the inelastic peaks measured with IXS, ${ }^{27,31,35}$ at least in the low- $Q$ range. In the limit of small $Q$ it is as well the solution of the hydrodynamic equations. ${ }^{40}$

The measured spectra are fitted to the sum of a $\delta$ function for the elastic line and a DHO profile for the inelastic contribution, convoluted with the spectrometer resolution. Examples of bestfitting line shapes are plotted in Fig. 1 at the two temperatures.

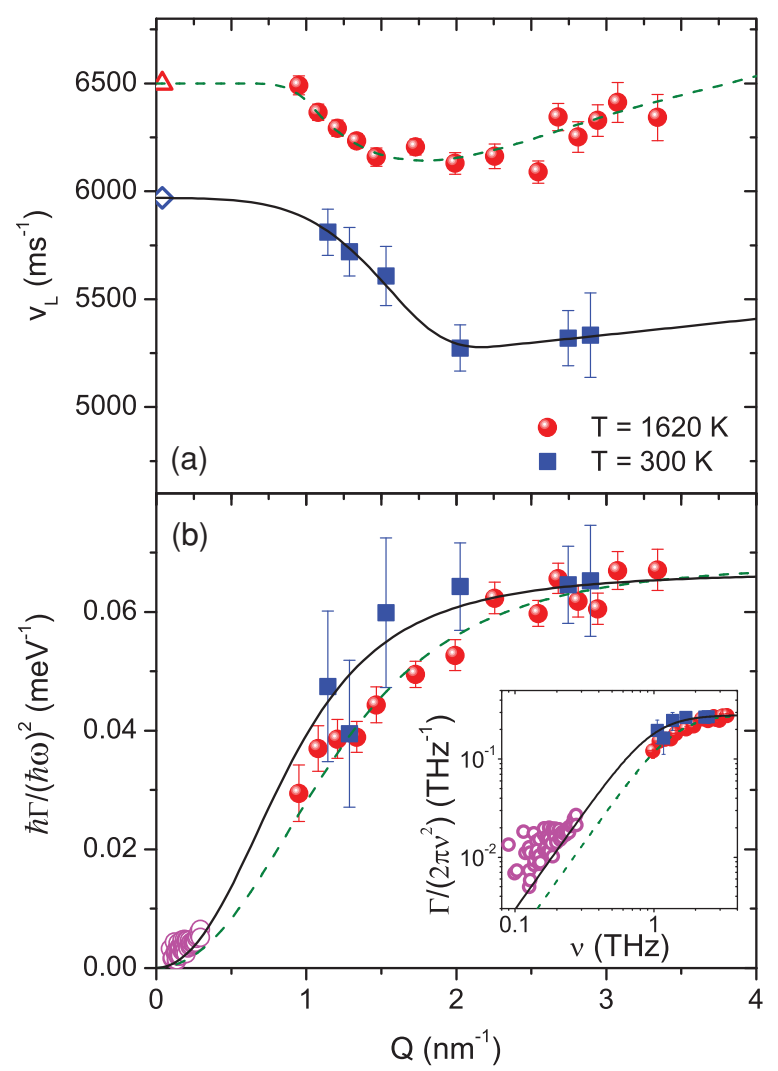

FIG. 2. (Color online) (a) $Q$ dependence of the apparent longitudinal sound velocity $v_{L}$ at the two indicated temperatures (the high-temperature data are taken from Ref. 23). The lines are fits to a phenomenological function describing the negative and positive dispersions. (b) Ratio between the damping $\Gamma$ and the square of the angular frequency as a function of the exchanged momentum $Q$. The continuous line (black) is the best-fitted curve to the room temperature points, while the dashed (olive) one is fitted to the high-temperature data ( $T=1620 \mathrm{~K}$, from Ref. 23). The curve is Eq. (5), describing the crossover from a $\Gamma \sim Q^{4}$ law for $Q \ll Q_{c}$ to a $\Gamma \sim Q^{2}$ law for $Q \gg Q_{c}$. The open circles (magenta) are data obtained with a tunneling-junction technique at $T=1 \mathrm{~K}$, from Ref. 41. Inset: The same quantity plotted as a function of frequency in log-log scale.

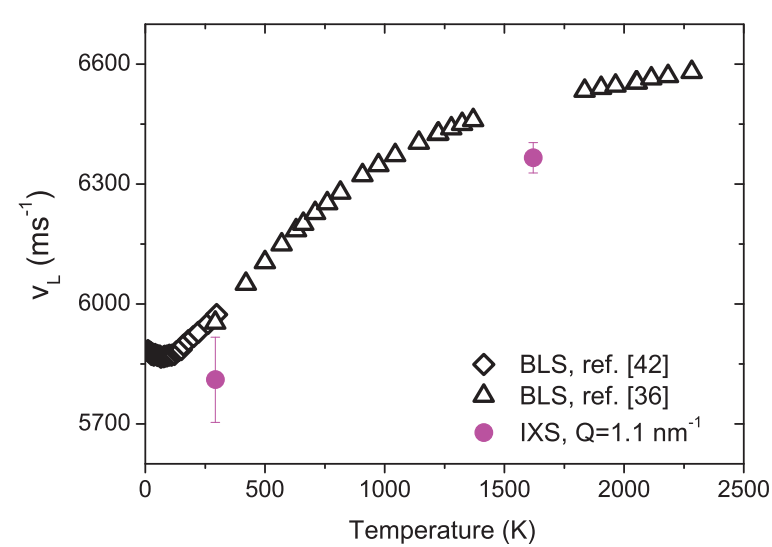

FIG. 3. (Color online) Longitudinal sound velocity as a function of temperature. Diamonds ${ }^{42}$ and triangles ${ }^{36}$ (black): BLS data, circles (magenta): IXS data at a wave vector $Q=1.1 \mathrm{~nm}^{-1}$.

\section{RESULTS AND DISCUSSION}

\section{A. Dispersion and damping}

The parameters extracted from the data analysis are plotted in Fig. 2. The upper panel of the figure shows the apparent longitudinal sound velocity $v_{L}=\Omega / Q$, while the lower panel presents the quantity $\Gamma / \hbar \omega^{2}$. In the low-wave-vector range the sound velocity, at both temperatures, is affected by a negative dispersion effect, followed by a positive dispersion at higher $Q$ 's. The negative dispersion has been discussed previously ${ }^{23}$ and it has been observed also in other systems. ${ }^{22,25}$

The data at room temperature indicate that $v_{L}$ increases markedly with increasing temperature. This variation is compatible with the temperature change of $v_{L}$ in the low-wavevector limit explored by BLS; ${ }^{36}$ see also Fig. 3. The BLS data are shown as open points in Fig. 2. The figure shows that the sound velocity in the terahertz range is close to but always lower than its low-frequency limit. This observation is in disagreement with a recent evaluation ${ }^{20}$ of the infinitefrequency sound velocity $v_{\infty}$, based on low-frequency BLS data, which is used to discuss the temperature evolution of the BP. There the authors estimate $v_{\infty}$ to be higher than the low-frequency value.

The temperature dependence of the longitudinal sound velocity is plotted in Fig. 3, where the BLS data are compared to our present IXS measurements at a wave vector $Q=$ $1.1 \mathrm{~nm}^{-1}$. The figure shows the characteristic increase of $v_{L}$ with temperature, which is due to the stiffening of the elastic constants as the temperature is increased.

Concerning the damping parameter $\Gamma$ [see Fig. 2(b)], the present data, confirming the result of the high temperature study, show a behavior which is not a simple $\Gamma \propto Q^{2}$ law, as found in the previous experiments. ${ }^{27-30}$ On the contrary, the damping undergoes a transition from $\Gamma \propto Q^{4}$ in the low- $Q$ range to $\Gamma \propto Q^{2}$ at high $Q$ 's (see Ref. 23 for a thorough discussion on this point). This behavior, best visible in the high-temperature data, is detectable also at room temperature. The $Q$ dependence of the sound attenuation coefficient $\Gamma$ can be phenomenologically described by the following function:

$$
\Gamma(Q)=a Q^{2}\left[1+\left(Q_{c} / Q\right)^{2 \delta}\right]^{-1 / \delta} .
$$


This function follows a $Q^{4}$ law for wave vectors much smaller than the crossover wave vector $Q_{c}$ and a $Q^{2}$ law in the opposite limit. The parameter $\delta$ defines the sharpness of the transition and is fixed to $\delta=1.5$. The best-fitting functions to the measured $\Gamma$ are plotted in Fig. 2(b). The crossover wave vectors are $Q_{c}=(1.41 \pm 0.06) \mathrm{nm}^{-1}$ at high temperature and $Q_{c}=$ $(1.1 \pm 0.1) \mathrm{nm}^{-1}$ at room temperature. Taking into account the temperature dependence of the sound velocity the transition takes place at the crossover energies $\hbar \omega_{c}=\hbar v_{L} Q_{c}=6.0 \mathrm{meV}$ at high temperature and $\hbar \omega_{c}=4.2 \mathrm{meV}$ at room temperature, thus coinciding exactly to the boson peak positions at the two temperatures (see Fig. 5). This coincidence between the crossover point and the boson peak position, which holds as a function of temperature, indicates a strong connection between the excess of vibrational modes and the change in slope of the sound attenuation.

The extrapolation to the low- $Q$ range of the $Q^{4}$ slope determined from the room temperature data is in good agreement with a low-temperature measurement of the sound attenuation obtained with a tunneling-junction technique. ${ }^{41}$ In contrast the extrapolation from the high-temperature IXS data is slightly displaced to higher $Q$ 's because of the $Q_{c}$ dependence on temperature, as can be better appreciated in the inset of Fig. 2(b), where the damping is plotted in log-log scale.

\section{B. Comparison with previous IXS measurements}

The IXS technique has been greatly improved in the last ten years thanks to better-performing undulators which deliver a higher x-ray flux, the use of low-noise detectors, and the overall improvement in the beamline stability. The effects of these developments on the data quality are particularly evident in the case of vitreous silica, which was one of the first systems to be investigated by means of IXS. The data collected at room temperature and at high temperature $(T=1620 \mathrm{~K}$ from the experiment described in a recent Letter ${ }^{23}$ ) are compared to those obtained in previous experiments, both at high temperature $\left[T=1050 \mathrm{~K},{ }^{27}\right.$ and $T=1370 \mathrm{~K}$ (Refs. 30, 32 and 33)] and at room temperature ${ }^{28}$ in Fig. 4. The figure shows both the apparent longitudinal sound velocity and the damping parameter divided by the wave vector squared. The quality of the new data is greatly improved compared to the old measurements, with uncertainties even an order of magnitude smaller. This improvement unveils the presence of elastic anomalies, negative dispersion of the sound velocity and change in slope of the sound attenuation around the BP energy, which were not detectable in the old data because of the noise.

It is worth underlining that the data in the left column of Fig. 4 can be compared to each other, although the experiments are performed at different temperatures. In fact, the variation of both the sound velocity (see Fig. 3) and the BP energy in the temperature range between 1000 and $1600 \mathrm{~K}$ is small with respect to the uncertainty of the old measurements.

It is also worth noting that the present result contradicts an earlier IXS determination ${ }^{32,33}$ of the temperature dependence of $v_{L}$. In that work the authors reported a much stronger temperature variation of $v_{L}$ in the IXS regime compared to the BLS one. However, at that time the sound velocity at

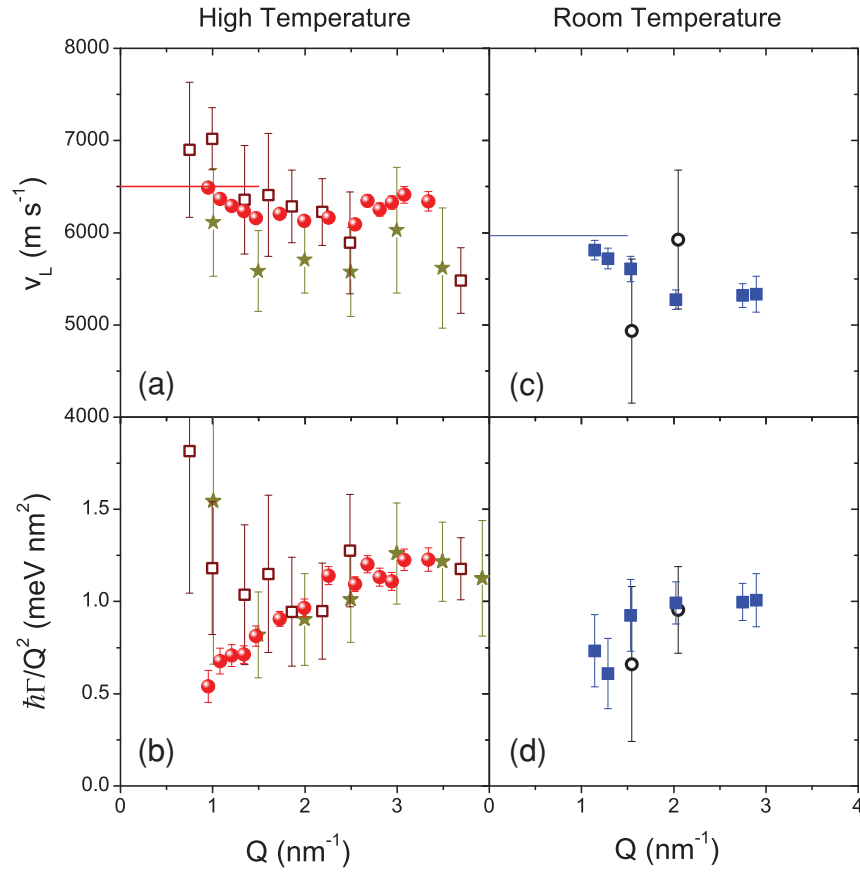

FIG. 4. (Color online) Comparison between the IXS data from the present work (room temperature) and from a recent Letter ( $T=1620 \mathrm{~K}$, Ref. 23 ) with previous studies from the literature. Left column: high temperature. Stars (dark yellow), $T=1050 \mathrm{~K}$, Ref. 27; open squares (wine), $T=1370 \mathrm{~K}$, Refs. 30, 32 and 33; points (red), $T=1620 \mathrm{~K}$, Ref. 23. (a) Longitudinal sound velocity at high temperature. The line (red) indicates the BLS value at $T=1620 \mathrm{~K}$. (b) Ratio between the sound attenuation and the wave vector squared at high temperature. Right column: room temperature data. Open circles (black), Ref. 28; full squares (blue), present work. (c) Longitudinal sound velocity at room temperature. The line (blue) indicates the BLS value at room temperature. (d) Ratio between the sound attenuation and the wave vector squared at room temperature.

room temperature was determined with a relative uncertainty of about 20\%, as shown in Fig. 4(c).

\section{Density of vibrational states estimate}

The connection between the measured elastic anomalies, dispersion of the sound velocity and change in slope of the attenuation, and the excess density of states at the boson peak can be explored in a more quantitative way. The density of vibrational states can be obtained from the trace of the resolvent operator, ${ }^{37,38}$ which can be computed in the wave vector base to be

$$
g(\omega)=-\frac{2 \omega}{3 N \pi} \sum_{Q} \operatorname{Im}\left[G_{L}\left(Q, \omega^{2}\right)+2 G_{T}\left(Q, \omega^{2}\right)\right] .
$$

Here $N$ is the number of atoms. Assuming a Debye distribution of wave vectors, the sum can be written as an integral, with the substitution $\frac{1}{N} \sum_{Q} \rightarrow \frac{3}{Q_{D}^{3}} \int_{0}^{Q_{D}} Q^{2} d Q$, where $Q_{D}=$ $6 \pi^{2} N / V$, with $V$ the volume, is the Debye wave vector. Based on results of molecular dynamics simulations of vitreous silica, ${ }^{47}$ we have assumed that, at the same frequency, the transverse self-energy coincides with the longitudinal one: $\Sigma_{T}\left(Q_{T}=\omega / v_{T}, \omega\right)=\Sigma_{L}\left(Q_{L}=\omega / v_{L}, \omega\right)$. The density 


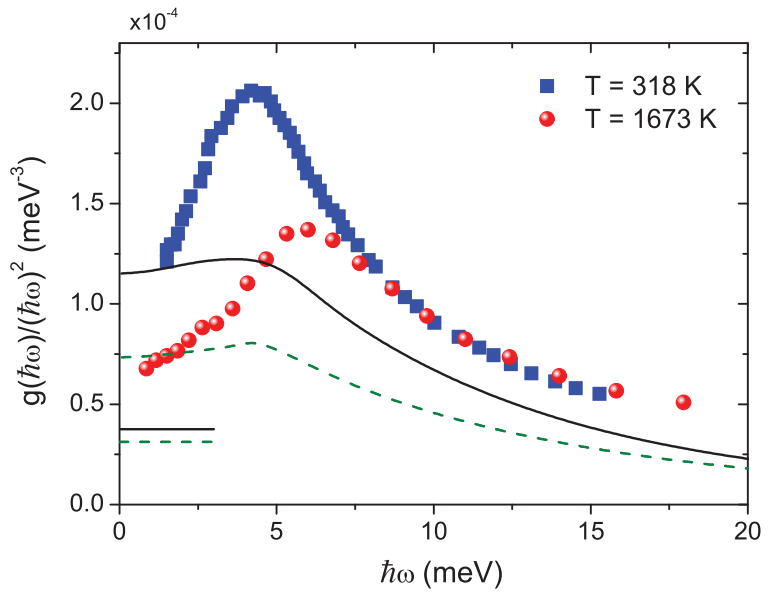

FIG. 5. (Color online) Temperature evolution of the reduced density of vibrational states $g(\omega) / \omega^{2}$ as measured with inelastic neutron scattering (Refs. 43 and 44). The solid and dashed lines are estimates of the acoustic contribution to the density of states, obtained by evaluating Eq. (6) as explained in the text (continuous black, $300 \mathrm{~K}$; dashed olive, $1620 \mathrm{~K}$ ). The horizontal lines are the Debye levels at the two temperatures (continuous black, $300 \mathrm{~K}$; dashed olive, $1620 \mathrm{~K})$.

of states is then estimated from the measured $v_{L}=v_{L}(Q)$ and $\Gamma=\Gamma_{L}(Q)$ functions, assuming for the self-energy the form of Eq. (4), leading to a DHO profile for the dynamic structure factor. The $Q$ dependences of $v_{L}$ and $\Gamma$ are fitted to appropriate phenomenological functions (see Fig. 2).

The computed $g(\omega) / \omega^{2}$ is plotted in Fig. 5 and compared to the density of states determined by neutron scattering. ${ }^{43,44}$ The calculated curves present a weak peak around $4 \mathrm{meV}$. However the curves are not able to reproduce the intensity of the boson peak and its shift with temperature.

We remark that the estimated density of states does not extrapolate to the Debye limit at low frequency (horizontal lines in the figure). The computed curves are in excellent agreement with the neutron data in the zero-frequency limit. This excess density comes from the strong increase of the damping parameter $\Gamma$ with $Q$. In fact, Eq. (6) reduces to the Debye density of states in the limit of zero sound attenuation $(\Gamma / \Omega \ll 1)$. The sound waves in a glass are always subject to a given amount of damping because of the structural disorder of the system. The finite damping induces an increase of $g(\omega) / \omega^{2}$ above the Debye level even at very low frequencies. Since the curves in Fig. 5 represent the acoustic contribution to the density of states we can state that the excess over the Debye level is of acoustic nature, at least in its low-frequency part. In the past other kinds of excitation were suggested to contribute to $g(\omega)$ in the low-frequency limit, such as tunneling in two-level systems. ${ }^{45,46}$

The failure of the model to describe the BP intensity has important implications for the nature of the boson peak. The result indicates that the main part of the BP is not associated with low- $Q$ acousticlike modes. This conclusion, however, does not exclude the possibility that the remaining part of the BP excess can come from acoustic modes at the border of the pseudo-Brillouin-zone boundary. In fact, our estimate of Eq. (6) assumes the acousticlike modes to be propagating at the apparent sound velocity up to the Debye wave vector $Q_{D}$, although strongly damped. The presence of nondispersing modes with energies around that of the boson peak has indeed been detected by means of IXS for wave vectors $Q>4 \mathrm{~nm}^{-1} \cdot{ }^{34,35}$ Excess vibrations were identified also from the analysis of neutron spectra in the form of librations of corner-sharing tetrahedra. ${ }^{1}$ The situation in vitreous silica appears thus noticeably different from that in other glasses, like glycerol ${ }^{22}$ and sorbitol, ${ }^{25}$ where a computation based on Eq. (6) well reproduced the BP shape.

\section{Comparison with the model by Schirmacher and co-workers}

In this section we will present a comparison of the experimental results with a recently proposed model for the vibrations in disordered systems. ${ }^{7,37}$ The model is based on the assumption that the disorder leads to microscopic random spatial fluctuations of the transverse elastic constants. The system is represented as a continuum medium with a shear modulus fluctuating in space around a mean value $G_{0}$ as $G(\boldsymbol{r})=G_{0}[1+\Delta \tilde{G}(\boldsymbol{r})]$ and a constant longitudinal modulus. The random function $\Delta \tilde{G}(\boldsymbol{r})$ is supposed to be Gaussian distributed with a variance proportional to $\gamma$. The parameter $\gamma$ describes the degree of disorder of the system. The model becomes unstable when $\gamma$ exceeds a critical value $\gamma_{c}$, which depends on the ratio $v_{L} / v_{T}$.

The complex self-energy of the model can be computed from a set of self-consistent equations. The only parameters entering the model are $\gamma$ and the ratio of the longitudinal and transverse sound velocities, $v_{L} / v_{T}$, at zero frequency. The knowledge of the self-energy allows a comparison to the experimentally accessible quantities, namely, the one-phonon dynamic structure factor and the density of vibrational states. In Fig. 6 we compare the frequency dependence of the model to the apparent sound velocity (top panel), the damping $\Gamma$ (middle panel), and the excess density of states (lower panel). All the quantities are plotted as functions of $\omega / \omega_{D}$, where $\omega_{D}=Q_{D} v_{D}$ is the Debye angular frequency, with $v_{D}$ the Debye velocity. It is worth noting that in the investigated temperature range the density variation of vitreous silica is negligible ${ }^{48}$ so that $Q_{D}$ can be considered temperature independent and $\omega_{D}$ depends on temperature through the sound velocity variation.

The model predictions are plotted for three values of the disorder parameter $\gamma$, while the ratio $v_{L} / v_{T}$ is fixed to the BLS value at room temperature. The model is able to reproduce qualitatively all the features observed in the experimental data. It shows a negative dispersion of the sound velocity at low frequency followed by a subsequent positive dispersion. The damping is characterized by a change in slope from a high power of frequency at low frequencies to an almost $\omega^{2}$ law at high frequencies. The model also presents a boson peak feature. Most importantly, the minimum in the sound velocity, the change in slope of the damping, and the excess in the reduced density of states take place all at the same frequency. As $\gamma$ is increased toward the critical value $\gamma_{c}$, these features shift to lower frequency.

The figure clearly shows that the model fails in a quantitative description of both the damping $\Gamma$ and the boson peak intensity, as pointed out in Ref. 49. The failure of the 


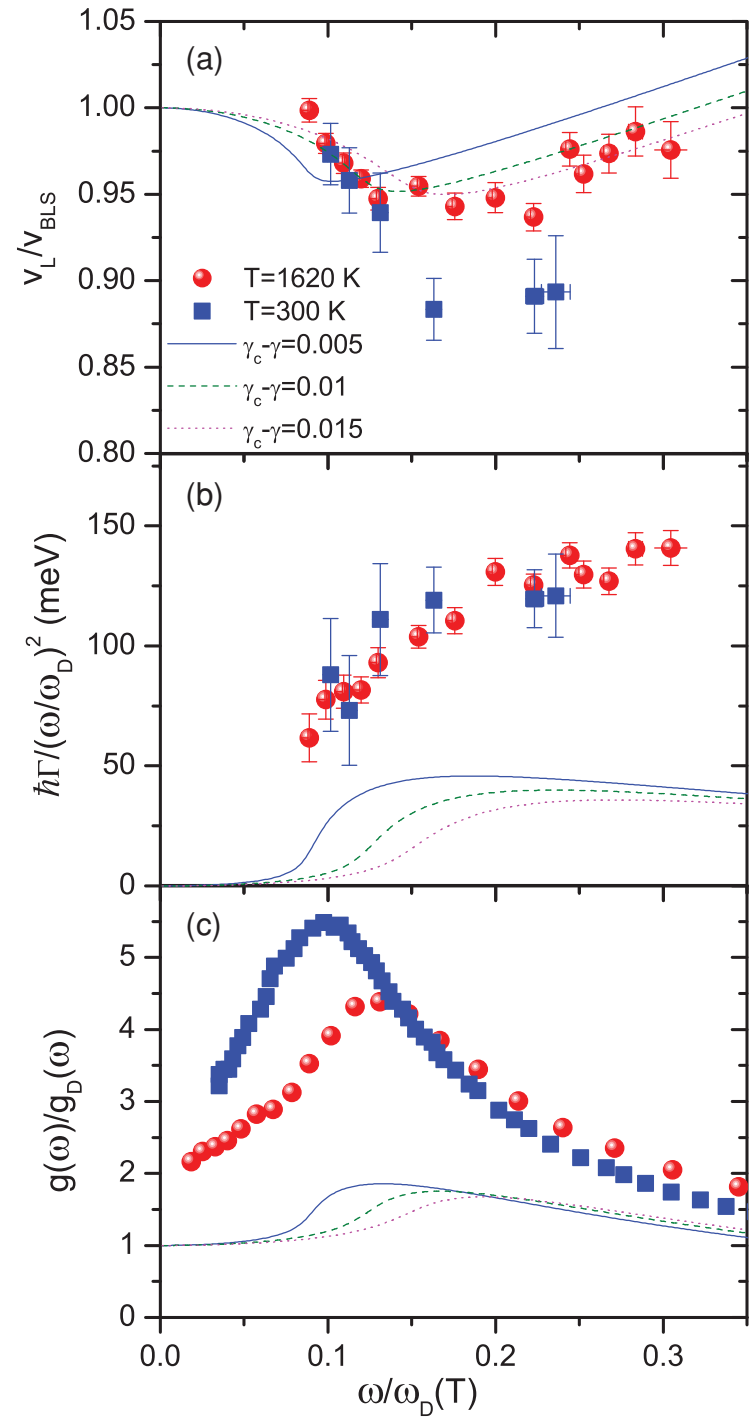

FIG. 6. (Color online) Apparent sound velocity, sound attenuation, and reduced excess of vibrational states plotted as a function of $\omega / \omega_{D}$ at the two probed temperatures. (a) shows the sound velocity divided by its BLS value $v_{\mathrm{BLS}}$. (b) presents the sound damping divided by the square of the frequency in units of the Debye frequency. In (c) the density of states is divided by the Debye density of states, $g_{D}(\omega)=3 \omega^{2} / \omega_{D}^{3}$, at the studied temperature. The curves are the prediction of the model by Schirmacher and co-workers (Refs. 7 and 37) for the three indicated values of the disorder parameter $\gamma$. The ratio $v_{L} / v_{T}$ is fixed to the room temperature BLS value and corresponds to a critical disorder parameter $\gamma_{c}=0.1815$.

model in accounting for the measured sound attenuation can be tentatively explained by taking into account that it is a model on the continuum. It can be viewed as a limiting case of a lattice model with fluctuating elastic constants. The absence of topological disorder may induce a smaller damping of the sound waves than in a real system. A small damping is also reflected in a small excess density of states, as explained in Ref. 37.

It is important to note that the model curves for all the three plotted quantities are unchanged if $v_{L} / v_{T}$ is varied according to the temperature dependence of the sound velocity in the
BLS regime. In other words, the model scales with the Debye frequency $\omega_{D}(T)$ if the disorder parameter $\gamma$ is kept fixed. On the contrary the experimental data show some temperature dependence beyond the temperature change of $\omega_{D}$. Most noticeably the BP does not follow the transformation of the elastic continuum medium, described by the Debye frequency. Both its position and its intensity undergo a variation with temperature which is stronger than the variation of $\omega_{D}(T)$. The model parameter $\gamma$ does not have a precise connection with temperature. However, the comparison with the experimental points suggests that it should increase as the temperature is lowered, indicating that the shear modulus distribution gets broader and broader as the system is cooled from the liquid down to the glassy state.

\section{CONCLUSIONS}

We have presented IXS data of vitreous silica at room temperature. From the comparison with data from a previous experiment ${ }^{23}$ we have determined the temperature dependence of the apparent sound velocity which, in the low-wavevector range, follows that measured by means of BLS at gigahertz frequencies. The room temperature points confirm the presence of elastic anomalies at frequencies corresponding to the excess of modes at the BP. In particular, the sound velocity shows a negative dispersion and the sound attenuation undergoes a transition from a dependence proportional to $Q^{4}$ to one proportional to $Q^{2}$. The transition takes place at a frequency which coincides with the BP frequency and is strongly temperature dependent. This variation is greater than the temperature change of the Debye frequency so that the elastic medium modification does not account for the BP variation.

The knowledge of the wave vector dependence of the frequency and damping of the acousticlike modes allows for a computation of their contribution to the density of vibrational states. The result indicates the BP maximum to be mainly due to nondispersive excitations. These modes can be associated with the low-frequency peak that appears in the IXS spectra at higher wave vectors. ${ }^{34,35}$ The failure of a simple Debye approach to describe the temperature dependence of the BP also supports the conclusion that other excitations accumulate in frequency at the BP.

A different behavior is found in the glycerol ${ }^{22}$ and sorbitol ${ }^{25}$ glasses, where the elastic anomalies appear to be responsible for the BP excess. This indicates that two mechanisms give rise to a piling up of vibrational states at the BP. One is the presence of a negative dispersion of the sound velocity and the corresponding change in slope of the sound attenuation. The other mechanism is the growing intensity of a branch of nondispersing excitations located close to the energy of the BP. These two mechanisms are probably not independent of one another. In fact the first mechanism, the one associated with the elastic anomalies, is reflected in a kink in the frequency dependence of the self-energy. This peculiar shape of the selfenergy gives rise to a second peak in the dynamic structure factor, just at the same energy as the kink, coinciding with the position of the BP and of the change in slope of the sound attenuation. This is in qualitative agreement with the model 
of Schirmacher and co-workers, where a second feature in the dynamic structure factor can be detected, in both its version on the continuum and that on the lattice. ${ }^{50}$ This second peak is even more evident in the version of the model with vector vibrations, studied by Taraskin and Elliott. ${ }^{51}$

The observed difference among the studied systems seems thus to reside in the relative weight of the two mechanisms in determining the BP intensity. The branch of nondispersing excitations is particularly intense in the case of vitreous silica $^{34,35}$ and represents an important part of the BP. In systems like glycerol and sorbitol the contribution to the BP excess of this feature, if present, is negligible and the softening of the sound waves dominates the BP intensity.

\section{ACKNOWLEDGMENT}

The authors would like to thank W. Schirmacher for fruitful discussions about his model. *giacomo.baldi@cnr.it

${ }^{1}$ U. Buchenau, N. Nücker, and A. J. Dianoux, Phys. Rev. Lett. 53, 2316 (1984).

${ }^{2}$ R. C. Zeller and R. O. Pohl, Phys. Rev. B 4, 2029 (1971).

${ }^{3}$ G. Monaco and S. Mossa, Proc. Natl. Acad. Sci. USA 106, 16907 (2009).

${ }^{4}$ D. A. Parshin, Fiz. Tverd. Tela (Leningrad) 36, 1809 (1994) [Phys. Solid State 36, 991 (1994)].

${ }^{5}$ W. Götze and M. R. Mayr, Phys. Rev. E 61, 587 (2000).

${ }^{6}$ W. Schirmacher, G. Diezemann, and C. Ganter, Phys. Rev. Lett. 81, 136 (1998).

${ }^{7}$ W. Schirmacher, Europhys. Lett. 73, 892 (2006).

${ }^{8}$ T. S. Grigera, V. Martin-Mayor, G. Parisi, and P. Verrocchio, Nature (London) 422, 289 (2003).

${ }^{9}$ C. Ganter and W. Schirmacher, Phys. Rev. B 82, 094205 (2010).

${ }^{10}$ A. Wischnewski, U. Buchenau, A. J. Dianoux, W. A. Kamitakahara, and J. L. Zarestky, Phys. Rev. B 57, 2663 (1998).

${ }^{11}$ S. Caponi, A. Fontana, F. Rossi, G. Baldi, and E. Fabiani, Phys. Rev. B 76, 092201 (2007).

${ }^{12}$ Y. Inamura, M. Arai, T. Otomo, N. Kitamura, and U. Buchenau, Physica B 284-288, 1157 (2000).

${ }^{13}$ M. Zanatta, G. Baldi, S. Caponi, A. Fontana, E. Gilioli, M. Krish, C. Masciovecchio, G. Monaco, L. Orsingher, F. Rossi, G. Ruocco, and R. Verbeni, Phys. Rev. B 81, 212201 (2010).

${ }^{14}$ A. Monaco, A. I. Chumakov, G. Monaco, W. A. Crichton, A. Meyer, L. Comez, D. Fioretto, J. Korecki, and R. Rüffer, Phys. Rev. Lett. 97, 135501 (2006).

${ }^{15}$ L. Orsingher, A. Fontana, E. Gilioli, G. Carini, G. Carini, G. Tripodo, T. Unruh, and U. Buchenau, J. Chem. Phys. 132, 124508 (2010).

${ }^{16}$ K. Niss, B. Begen, B. Frick, J. Ollivier, A. Beraud, A. Sokolov, V. N. Novikov, and C. Alba-Simionesco, Phys. Rev. Lett. 99, 055502 (2007).

${ }^{17}$ L. Hong, B. Begen, A. Kisliuk, C. Alba-Simionesco, V. N. Novikov, and A. P. Sokolov, Phys. Rev. B 78, 134201 (2008).

${ }^{18}$ S. Caponi, S. Corezzi, D. Fioretto, A. Fontana, G. Monaco, and F. Rossi, Phys. Rev. Lett. 102, 027402 (2009).

${ }^{19}$ A. Monaco, A. I. Chumakov, Y.-Z. Yue, G. Monaco, L. Comez, D. Fioretto, W. A. Crichton, and R. Rüffer, Phys. Rev. Lett. 96, 205502 (2006).

${ }^{20}$ B. Rufflé, S. Ayrinhac, E. Courtens, R. Vacher, M. Foret, A. Wischnewski, and U. Buchenau, Phys. Rev. Lett. 104, 067402 (2010).

${ }^{21}$ G. Baldi, A. Fontana, G. Monaco, L. Orsingher, S. Rols, F. Rossi, and B. Ruta, Phys. Rev. Lett. 102, 195502 (2009).

${ }^{22}$ G. Monaco and V. M. Giordano, Proc. Natl. Acad. Sci. USA 106, 3659 (2009).
${ }^{23}$ G. Baldi, V. M. Giordano, G. Monaco, and B. Ruta, Phys. Rev. Lett. 104, 195501 (2010).

${ }^{24}$ G. Baldi, V. M. Giordano, G. Monaco, and B. Ruta, J. Non-Cryst. Solids 357, 538 (2011).

${ }^{25}$ B. Ruta, G. Baldi, V. M. Giordano, L. Orsingher, S. Rols, F. Scarponi, and G. Monaco, J. Chem. Phys. 133, 041101 (2010).

${ }^{26}$ M. Foret, E. Courtens, R. Vacher, and J.-B. Suck, Phys. Rev. Lett. 77, 3831 (1996).

${ }^{27}$ P. Benassi, M. Krisch, C. Masciovecchio, V. Mazzacurati, G. Monaco, G. Ruocco, F. Sette, and R. Verbeni, Phys. Rev. Lett. 77, 3835 (1996).

${ }^{28}$ C. Masciovecchio, G. Ruocco, F. Sette, P. Benassi, A. Cunsolo, M. Krisch, V. Mazzacurati, A. Mermet, G. Monaco, and R. Verbeni, Phys. Rev. B 55, 8049 (1997).

${ }^{29}$ F. Sette, M. Krisch, C. Masciovecchio, G. Ruocco, and G. Monaco, Science 280, 1550 (1998).

${ }^{30}$ G. Ruocco, F. Sette, R. Di Leonardo, D. Fioretto, M. Krisch, M. Lorenzen, C. Masciovecchio, G. Monaco, F. Pignon, and T. Scopigno, Phys. Rev. Lett. 83, 5583 (1999).

${ }^{31}$ O. Pilla, A. Cunsolo, A. Fontana, C. Masciovecchio, G. Monaco, M. Montagna, G. Ruocco, T. Scopigno, and F. Sette, Phys. Rev. Lett. 85, 2136 (2000).

${ }^{32}$ T. Scopigno, Laurea thesis, L'Aquila University, Italy, 1998.

${ }^{33}$ C. Masciovecchio, V. Mazzacurati, G. Monaco, G. Ruocco, T. Scopigno, F. Sette, P. Benassi, A. Cunsolo, A. Fontana, M. Krisch, A. Mermet, M. Montagna, F. Rossi, M. Sampoli, G. Signorelli, and R. Verbeni, Philos. Mag. B 79, 2013 (1999).

${ }^{34}$ B. Ruzicka, T. Scopigno, S. Caponi, A. Fontana, O. Pilla, P. Giura, G. Monaco, E. Pontecorvo, G. Ruocco, and F. Sette, Phys. Rev. B 69, 100201(R) (2004).

${ }^{35}$ G. Baldi, V. M. Giordano, G. Monaco, F. Sette, E. Fabiani, A. Fontana, and G. Ruocco, Phys. Rev. B 77, 214309 (2008).

${ }^{36}$ A. Polian, Dung Vo-Thanh, and P. Richet, Europhys. Lett. 57, 375 (2002).

${ }^{37}$ W. Schirmacher, G. Ruocco, and T. Scopigno, Phys. Rev. Lett. 98, 025501 (2007)

${ }^{38}$ E. N. Economou, Green's Functions in Quantum Physics 2nd ed. (Springer-Verlag, New York, 1983).

${ }^{39}$ S. Ciliberti, T. S. Grigera, V. Martin-Mayor, G. Parisi, and P. Verrocchio, J. Chem. Phys. 119, 8577 (2003).

${ }^{40}$ J. P. Boon and S. Yip, Molecular Hydrodynamics (McGraw-Hill, New York, 1980).

${ }^{41}$ W. Dietsche and H. Kinder, Phys. Rev. Lett. 43, 1413 (1979).

${ }^{42}$ R. Vacher, J. Pelous, F. Plicque, and A. Zarembowitch, J. Non-Cryst. Solids 45, 397 (1981). 
${ }^{43}$ A. Wischnewski, U. Buchenau, A. J. Dianoux, W. A. Kamitakahara, and J. L. Zarestky, Phys. Rev. B 57, 2663 (1998).

${ }^{44}$ A. Fontana, R. Dell'Anna, M. Montagna, F. Rossi, G. Viliani, G. Ruocco, M. Sampoli, U. Buchenau, and A. Wischnewski, Europhys. Lett. 47, 56 (1999).

${ }^{45}$ G. Winterling, Phys. Rev. B 12, 2432 (1975).

${ }^{46} \mathrm{~N}$. Theodorakopoulos and J. Jckle, Phys. Rev. B 14, 2637 (1976).
${ }^{47}$ S. N. Taraskin and S. R. Elliott, Phys. Rev. B 61, 12031 (2000).

${ }^{48}$ R. Brückner, J. Non Cryst. Solids 5, 123 (1970).

${ }^{49}$ B. Rufflé, D. A. Parshin, E. Courtens, and R. Vacher, Phys. Rev. Lett. 100, 015501 (2008).

${ }^{50}$ W. Schirmacher, G. Diezemann, and C. Ganter, Physica B 263-264, 160 (1999).

${ }^{51}$ S. N. Taraskin and S. R. Elliott, J. Phys.: Condens. Matter 14, 3143 (2002). 